\title{
Perceptual interference decays over short unfilled intervals
}

\author{
MATTHEW D. SCHULKIND \\ Duke University, Durham, North Carolina
}

\begin{abstract}
The perceptual interference effect refers to the fact that object identification is directly related to the amount of information available at initial exposure. The present article investigated whether perceptual interference would dissipate when a short, unfilled interval was introduced between exposures to a degraded object. Across three experiments using both musical and pictorial stimuli, identification performance increased directly with the length of the unfilled interval. Consequently, significant perceptual interference was obtained only when the interval between exposures was relatively short ( $<500$ msec for melodies; $<300$ msec for pictures). These results are consistent with explanations that attribute perceptual interference to increased perceptual noise created by exposures to highly degraded objects. The data also suggest that perceptual interference is mediated by systems that are not consciously controlled by the subject and that perceptual interference in the visual domain decays more rapidly than perceptual interference in the auditory domain.
\end{abstract}

The ability to identify an object varies directly with the amount of information present at initial exposure. This general effect has been given several names, including the perceptual interference effect and the Bruner-Potter ef$f e c t$, after the researchers credited with the first empirical demonstration of the phenomenon. Bruner and Potter (1964) had subjects identify blurred pictures that were gradually brought into focus. Images were initially exposed at one of three levels of blur (very, moderately, and lightly blurred), but all were eventually brought to the same level of focus. The initial level of blur was inversely related to identification performance: The more blurred the initial presentation, the less likely subjects were to identify the stimulus, even though all photographs were ultimately seen at the same level of blur.

More recent investigations have used different procedures and stimuli but have also found a direct relationship between identification performance and the amount of information available at initial exposure. For example, rather than presenting blurred photographic images, Snodgrass and her colleagues (Luo \& Snodgrass, 1994; Snodgrass \& Hirshman, 1991) used fragmented versions of line drawings taken from the Snodgrass and Vanderwort (1980) norms. The line drawings were presented in one of two ways. Fixed presentation involved a single exposure to a

I thank David Rubin, Tamara Rahhal, and Dave Irwin for helpful comments on an earlier draft. Gordon Logan provided a great deal of advice on preparing the manuscript. Joan Gay Snodgrass, Mark Schmuckler, and Timothy McNamara also offered helpful comments. Special thanks to Robert Gordon for writing the program to run Experiment 3. Portions of this work were included in a doctoral dissertation submitted to the Graduate School of Duke University. Correspondence should be addressed to M. D. Schulkind, who is currently at the Department of Psychology, Amherst College, Amherst, MA 01002 (e-mail: mdschulkind@) amherst.edu). moderately fragmented image. Ascending presentation involved four exposures to the same base stimulus. The level of fragmentation in the ascending condition was gradually decreased in such a way that the last exposure in the ascending condition was identical to the one and only exposure in the fixed condition. Despite the fact that both conditions ultimately displayed the same information, the fixed condition yielded better identification performance than the ascending condition. Notably, the identification advantage for fixed presentation obtained even though the subjects received multiple exposures during ascending, but not fixed, presentation.

The present study was designed to investigate the boundary conditions for the emergence of perceptual interference by examining the effect of introducing an unfilled interval between successive exposures in an ascending sequence. In a typical perceptual interference experiment, the target object is never removed from view. For example, in Bruner and Potter's (1964) experiment, the target object was continuously exposed and slowly brought into focus. In more recent experiments, which have compared fixed and ascending presentation modes, the multiple exposures in the ascending sequence usually followed one another immediately. One exception to this rule is an experiment conducted by Snodgrass and Hirshman (1991) in which the target object was briefly removed from the screen in favor of a mathematical filler task. They found that introducing a single-digit addition problem between exposures in the ascending sequence eliminated perceptual interference (see Frederiksen, 1969 , for a similar effect with auditory presentation of words). That is, performance in the ascending condition did not differ from performance in the fixed condition. Thus, unlike most filler tasks, interpolating a simple arithmetic problem into a perceptual series had the paradoxical effect of improving identification performance. However, 
it is unclear whether the interference effect was eliminated by the filler task itself or by the removal of the target object during the presentation of the task. In other words, did the filler task eliminate the activation responsible for perceptual interference, or did this activation merely decay across the delay that the filler task introduced? The answer to this question is important because it will help illuminate the mechanism underlying the effect. If a filler task is needed to eliminate perceptual interference, one might conclude that working memory structures such as the phonological loop or visuospatial sketchpad were involved in the effect. In contrast, if perceptual interference dissipates across an unfilled interval, it would be more likely that perceptual interference was mediated by other mechanisms and/or processes.

In addition to identifying an important boundary condition for observing perceptual interference, introducing a short, unfilled delay into an ascending sequence will also help distinguish between two competing explanations for the effect. The blocking hypothesis attributes perceptual interference to the adoption of incorrect hypotheses regarding the identity of the to-be-identified object (Bruner \& Potter, 1964). Once adopted, these distractor objects are very difficult to dismiss and therefore "block" retrieval of the target. As the interval between exposures to the target increases, so does the opportunity to generate incorrect guesses. Therefore, the blocking hypothesis predicts that perceptual interference will increase with the length of an unfilled interval.

The competitive activation hypothesis (Snodgrass \& Hirshman, 1991) holds that perceptual interference is caused by the activation of perceptual units associated with distractor objects. Initial exposures to an object contain elements common to the target and to one or more distractors. Activation from these common elements spreads to perceptual units of the target and the associated distractors. The activation of nontarget units creates noise in the system that interferes with identification. One would assume that this distracting activation would begin to dissipate as soon as the target becomes unavailable (e.g., is removed from the computer screen). Therefore, as the length of an unfilled interval between exposures increases, the distracting activation should decrease, and consequently so should the likelihood of observing perceptual interference.

The experiments described below used the basic methodology of recent work in the literature (Luo \& Snodgrass, 1994; Peynircioğlu, 1987; Peynircioğlu \& Watkins, 1986; Snodgrass \& Hirshman, 1991) to examine the effect of introducing an unfilled interval into an ascending presentation sequence. The subjects identified objects presented in either a fixed (all information presented initially) or an ascending (information gradually revealed over the course of multiple trials) format. In addition to the pictorial stimuli used in previous work, the present study also examined perceptual interference for musical stimuli. In the music studies, the subjects were asked to identify familiar songs like "Frosty, the Snowman" on the basis of the first several notes. Familiar music was se- lected as the target stimulus for a variety of reasons. First, it would extend the generalizability of the perceptual interference effect to a new domain. More importantly, using musical stimuli would provide a nonarbitrary way of increasing information from one exposure to the next in the ascending sequence. For all melodies, the first presentation in the ascending sequence consisted of the first two notes. Each subsequent presentation added a single note (i.e., the second presentation consisted of the first three notes, the third presentation of the first four notes, etc.). The final presentation in the ascending condition contained the same number of notes as the single presentation in the fixed condition. Finally, using melodies eased the introduction of interstimulus intervals (ISIs) because each presentation had distinct temporal beginnings and endings as well as nonarbitrary durations.

\section{EXPERIMENT 1}

Experiment 1 was designed to address three questions regarding the perceptual interference effect. First, will identification performance vary directly (competitive activation) or inversely (blocking) with the length of a silent, unfilled interval introduced between successive exposures in an ascending sequence? Second, will an unfilled interval eliminate perceptual interference, or is some kind of filler task necessary? Third, will the perceptual interference effect generalize to musical stimuli?

These issues were addressed by contrasting identification performance across fixed and ascending presentation formats. The number of notes played for each song was selected to yield moderate levels of identification performance. The Appendix shows the number of notes heard for each of the 32 target songs along with the cumulative probability of identifying the song by that number of notes in other work (Schulkind, Posner, \& Rubin, 2000 ). In the present experiment, the fixed condition consisted of a single exposure to the first several notes of the target song. The ascending condition consisted of multiple presentations. The first presentation consisted of the first two notes. The second presentation consisted of the first three notes; the third presentation consisted of the first four notes, and so on. Notes were added one by one until the number of notes matched the number heard in the fixed condition. Thus, the number of notes heard in the final presentation of the ascending condition was identical to the number of notes heard in the single presentation in the fixed condition.

Successive exposures in the ascending sequence were separated by an experimenter-determined ISI that was set at one of three lengths: 50,500 , and $5,000 \mathrm{msec}$. These intervals were selected to represent equally spaced points on a logarithmic scale. The lower boundary was selected because $50 \mathrm{msec}$ has been cited as a rough estimate of the time listeners need to hear two elements as consecutive instead of simultaneous (Handel, 1993). Further, this delay was easily perceived by the subjects in the pilot studies. The upper boundary was selected because it was approximately the duration of the longest song in the fixed 
condition ("Yesterday," 5,512 msec). Unlike previous research (Frederiksen, 1969; Snodgrass \& Hirshman, 1991), Experiment 1 used silent and unfilled ISIs; that is, they did not include a filler or judgment task.

In terms of the present experiment, perceptual interference would be observed if identification performance in the ascending condition was significantly worse than identification performance in the fixed condition. The competitive activation hypothesis predicts that there will be a direct relationship between identification performance in the ascending condition and the length of the ISI. In other words, it predicts that perceptual interference will be observed only at short ISIs. The blocking hypothesis, on the other hand, predicts that identification performance will be inversely related to ISI length because shorter ISIs will not provide much time for the generation of incorrect hypotheses. In other words, it predicts that perceptual interference will be observed only at longer ISIs.

\section{Method}

Subjects. Forty undergraduate volunteers received partial credit toward a course requirement in exchange for their participation and were randomly assigned to one of four counterbalancing orders. No subjects were music majors.

Design. A one-way within-subjects design was used. Presentation style (fixed, ascending - 50-msec ISI; ascending-500-msec ISI ascending-5,000-msec ISI) was the only independent variable.

Materials. The 32 songs used in the experiment are listed in the Appendix. All songs were pretested by a group of 97 undergraduates. A majority of the pilot subjects claimed that they "easily knew most of the words to the song." A subset of the notes from each song was selected by determining the number of notes that led to moderate identification performance $(40 \%-65 \%)$ in previous work (Schulkind et al., 2000). The Appendix lists the number of notes heard for each song. For example, the subjects heard the first four notes of "Happy Birthday to You." In the fixed condition, the subjects received one exposure consisting of the selected notes for that song (e.g., the first four notes of "Happy Birthday to You"). On average, songs in the fixed condition consisted of 6.32 notes and had an average duration of $2.78 \mathrm{sec}$; the average duration per note was approximately $439 \mathrm{msec}$.

For ascending presentation, the subjects received multiple exposures to the melody. The first exposure consisted of the first two notes. Each subsequent exposure added one additional note; that is, the second exposure consisted of the first three notes, the third exposure consisted of the first four notes, and so on. The final exposure in the ascending condition always contained the same number of notes as the single presentation in the fixed condition. Each exposure in the ascending condition was separated by a silent interval of one of three lengths: 50,500 , or $5,000 \mathrm{msec}$
The requisite number of notes for each song was transferred from a published sheet music arrangement to a MacIntosh IIsi computer; the computer controlled the timing of stimulus presentations. Songs were presented in the same random order to each subject. Presentation format was assigned across four counterbalancing orders in such a way that, across the experiment, each song appeared an equal number of times in each format.

Procedure. The subjects were told that they would be asked to identify familiar songs on the basis of the first several notes. Fixed presentation was explained and illustrated by an example ("Old McDonald Had a Farm"). Next, ascending presentation was explained and illustrated with the same song. The ISI for the example was $500 \mathrm{msec}$; the subjects were informed that the length of the "delay" (ISI) would vary from song to song.

The subjects were instructed to respond orally using one of the following five responses: (1) "I have no idea,"(2) "It sounds familiar but I am not certain enough to make a guess,"(3) "I am not sure but it might be

or (5) "I am sure that it is__." The five response options " (4) "I am pretty sure that it is were printed on a sheet of paper placed in front of the subject. Responses 3-5 were all considered correct if they included either the appropriate song title or at least five consecutive lyrics from the song. For example, "... had a very shiny nose" would have been considered a correct response for "Rudolph, the Red-Nosed Reindeer." All other responses were considered incorrect. The response options also provided a 5-point scale of confidence ratings with each response representing a different level of confidence. However, analyses of these data yielded no significant differences, so they will not be discussed further. The subjects were given a maximum of $10 \mathrm{sec}$ to make a response. The experimenter recorded all responses and monitored the length of the response period with a stopwatch.

At the end of the experiment, the subjects were given a list of the experimental stimuli and asked whether there were any songs that they did not know. Data for songs that the subjects claimed not to know (less than $2.5 \%$ of the data) were eliminated.

\section{Results}

Every analysis in the article was conducted twice, once with subjects $\left(F_{1}\right)$ and once with songs $\left(F_{2}\right)$ as the unit of analysis. Because none of the data met the assumption of sphericity, every analysis was conducted using the procedures described by Hays (1994); alpha was set at .05 .

Mean percent correct identification for the fixed and three ascending ISI conditions was averaged over both subjects and songs. As can be seen in Table 1, identification performance increased directly with ISI length. A trend analysis revealed a significant linear trend when the ISI data were plotted using both linear $\left[F_{1}(1,39)=\right.$ $\left.11.59, M S_{\mathrm{e}}=.026 ; F_{2}(1,31)=6.68, M S_{\mathrm{e}}=.035\right]$ and

Table 1

Mean Percent Correct, and Standard Errors, by Presentation Condition

\begin{tabular}{|c|c|c|c|c|c|c|c|c|}
\hline \multirow[b]{3}{*}{ Condition } & \multicolumn{4}{|c|}{ Experiment 1} & \multicolumn{4}{|c|}{ Experiment 2} \\
\hline & \multicolumn{2}{|c|}{ Subjects } & \multicolumn{2}{|c|}{ Songs } & \multicolumn{2}{|c|}{ Subjects } & \multicolumn{2}{|c|}{ Songs } \\
\hline & $M$ & $S E$ & $M$ & $S E$ & $M$ & $S D$ & $M$ & $S E$ \\
\hline 50 & 52.33 & $3.92 *$ & 52.42 & $3.87^{*}$ & - & - & - & - \\
\hline 150 & - & - & & & 54.02 & $3.90^{*}$ & 53.85 & $4.15^{*}$ \\
\hline 300 & - & - & & & 56.44 & $3.54^{*}$ & 56.63 & $3.57^{*}$ \\
\hline 500 & 58.57 & 3.58 & 58.14 & 3.43 & - & - & - & - \\
\hline 600 & - & - & - & - & 60.03 & 3.64 & 59.86 & 3.34 \\
\hline 5,000 & 64.54 & 3.33 & 64.53 & 3.66 & - & - & - & - \\
\hline Fixed & 63.95 & 3.02 & 64.29 & 3.83 & 68.26 & 3.49 & 68.23 & 3.15 \\
\hline
\end{tabular}


logarithmic $\left[F_{1}(1,39)=8.96, M S_{\mathrm{e}}=.027 ; F_{2}(1,31)=\right.$ $\left.6.12, M S_{\mathrm{e}}=.032\right]$ coordinates. This pattern is consistent with the competitive activation framework, but not with the blocking hypothesis.

Perceptual interference was measured by calculating difference scores between the fixed condition and each level of ISI (i.e., fixed vs. ascending, for each ISI level). With the use of this methodology, significant perceptual interference would be observed when the average difference score was significantly greater than zero. The Bonferroni adjustment was used to maintain alpha at .05 . Significant perceptual interference was observed at the 50 -msec ISI $\left[t_{1}(39)=2.85, M S_{\mathrm{e}}=.033 ; t_{2}(31)=2.35\right.$, $\left.M S_{\mathrm{e}}=.041, .05<p<.10\right]$, but not at either the $500-\mathrm{msec}$ ISI $\left[t_{1}(39)=1.48, M S_{\mathrm{e}}=.026 ; t_{2}(31)=1.39, M S_{\mathrm{e}}=\right.$ $.031]$ or the $5,000-\mathrm{msec}$ ISI $\left[t_{1}(39)=-0.15, M S_{\mathrm{e}}=\right.$ $\left..032 ; t_{2}(31)=-0.05, M S_{\mathrm{e}}=.036\right]$. The $p$ values for all nonsignificant comparisons were greater than .40 .

\section{Discussion}

The results of this experiment suggest that an unfilled interval is sufficient to eliminate perceptual interference. Although a filler task--such as Snodgrass and Hirshman's (1991) single-digit addition problem - will eliminate perceptual interference, it is not necessary.

The overall pattern of identification performance supports the competitive activation model over the blocking hypothesis. Identification performance was directly related to the length of the ISI. Further, significant perceptual interference was obtained only at the shortest ISI (with the effect being only marginally significant on the item analysis). Although the $500-\mathrm{msec}$ ISI resulted in lower overall performance than the fixed condition, this difference was not significant. Notably, there were only very small differences between the fixed and $5,000-\mathrm{msec}$ condition despite the fact that the 5,000-msec ISI afforded the listeners multiple exposures to the target and more time to attempt identification. On the basis of these considerations, one might have predicted a facilitative effect of very long ISIs, though none was observed.

\section{EXPERIMENT 2}

An alternative explanation for the results of Experiment 1 is possible. The $50-\mathrm{msec}$ ISI, which was the only delay that showed the typical perceptual interference effect, may have been too short to allow the subjects to determine when the melodic pattern was restarted. This explanation seems unlikely because no subjects reported having such difficulty during postexperimental interviews. In addition, if auditory masking occurred only at the 50-msec ISI, one would expect to observe a significant difference between the 50- and the 500-msec ISIs. However, this difference did not approach significance $\left[t_{1}(39)=1.51, M S_{\mathrm{e}}=.034, p>.13 ; t_{2}(31)=1.27, M S_{\mathrm{e}}=\right.$ $.032, p>.20]$. Therefore, it is unlikely that the subjects were unable to discriminate the melodic patterns at the 50 -msec ISI.
Still, it is important to replicate these findings both to eliminate this alternative interpretation and to gain a finer grasp of how much time is required for listeners to overcome perceptual interference. Thus, Experiment 2 utilized the same basic design as Experiment 1, changing only the lengths of the ISIs. Three new ISIs were chosen to approximately cover the range between 50 and $500 \mathrm{msec}$. The new ISIs were 150,300 , and $600 \mathrm{msec}$. Again, these intervals were selected to represent equally spaced points on a logarithmic scale.

\section{Method}

Subjects. Forty undergraduate volunteers received partial credit toward a course requirement in exchange for their participation and were randomly assigned to one of four counterbalancing orders. No subjects were music majors.

Design. The experiment was a one-way within-subjects design. Presentation style (fixed, ascending - 150-msec ISI; ascending300 -msec ISI; ascending - 600-msec ISI) was the only independent variable.

Materials. The materials were the same as those used in Experiment 1 , with the following changes. The ISIs used in ascending presentation were 150,300 , or $600 \mathrm{msec}$ in length. A new random order was constructed for the presentation of the songs; this order was the same for each subject. Four new counterbalancing orders were constructed in such a way that each song appeared in each presentation format an equal number of times across the experiment.

Procedure. The procedure was identical to that used in Experiment 1.

\section{Results}

Data for songs that subjects claimed not to know were eliminated; this accounted for less than $2.5 \%$ of the data. Mean percent correct identification for the fixed and three ascending ISI conditions were averaged over both subjects and songs. As can be seen in Table 1, identification performance increased directly with ISI length. However, unlike in Experiment 1, the test for the linear trend did not approach even marginal levels of significance (all $p \mathrm{~s}>.20$ ) using either linear $\left[F_{1}(1,39)=2.92, M S_{\mathrm{e}}=.025 ; F_{2}(1,31)\right.$ $\left.=2.33, M S_{\mathrm{e}}=.025\right]$ or logarithmic $\left[F_{1}(1,39)=2.71\right.$, $\left.M S_{\mathrm{e}}=.027 ; F_{2}(1,31)=2.51, M S_{\mathrm{e}}=.023\right]$ coordinates.

Perceptual interference was analyzed using the same method as in Experiment 1. Significant perceptual interference was observed at the 150 -msec $\left[t_{1}(39)=3.09\right.$, $\left.M S_{\mathrm{e}}=.042 ; t_{2}(31)=3.11, M S_{\mathrm{e}}=.034\right]$ and $300-\mathrm{msec}$ $\left[t_{1}(39)=3.47, M S_{\mathrm{e}}=.023 ; t_{2}(31)=2.92, M S_{\mathrm{e}}=.025\right]$ ISIs. At the $600-\mathrm{msec}$ ISI, the perceptual interference effect did not approach significance for the subject analysis $\left[t_{1}(39)=2.00, M S_{\mathrm{e}}=.034, p>.15\right]$, but was marginally significant for the item analysis $\left[t_{2}(31)=2.85\right.$, $\left.M S_{\mathrm{e}}=.033, .05<p<.10\right]$

\section{Discussion}

Comparisons of the three ascending conditions with the fixed condition revealed significant perceptual interference at the 150- and 300-msec ISIs. The analyses regarding the $600-\mathrm{msec}$ ISIs were mixed; the subject analysis yielded no perceptual interference effect, whereas the item analysis revealed a marginal effect. This closely conforms 
to the results of Experiment 1, in which performance with a 500-msec ISI was lower than, but not significantly different from, performance in the fixed condition. Thus, the results of the both experiments point to the same conclusion: The performance advantage generally shown for fixed presentation can be eliminated if a brief (approximately 500-msec), unfilled ISI is introduced between subsequent presentations in the ascending sequence. Again, these results are consistent with the competitive activation framework, but not the blocking hypothesis.

Although the results of the first two experiments were consistent with each other, the linear trend analyses were significant only in Experiment 1. The failure to obtain a significant effect in the Experiment 2 may have resulted from the restricted range of ISIs. However, combining the data from Experiments 1 and 2 reveals a very consistent pattern. As can be seen in Figure 1, which depicts combined data from Experiments 1 and 2, percent correct shared a linear relationship with the logarithm of the ISI duration; each doubling of the ISI increased identification performance by approximately $2 \%$. The regression line explains approximately $97 \%$ of the variance in percent correct. Thus, despite the lack of a significant trend in Experiment 2, it is clear that identification performance increased, rather than decreased, as the ISI became longer.

\section{EXPERIMENT 3}

Although the data relating melody identification and ISI were very convincing for the musical stimuli used in Experiments 1 and 2, a third experiment was conducted to determine whether the timing effect would generalize to visual stimuli. Although some perceptual interference research has used auditory stimuli (Blake \& Vanderplas, 1950; Frederiksen, 1967, 1969), most prior research has used visual materials. The results of the first two exper-

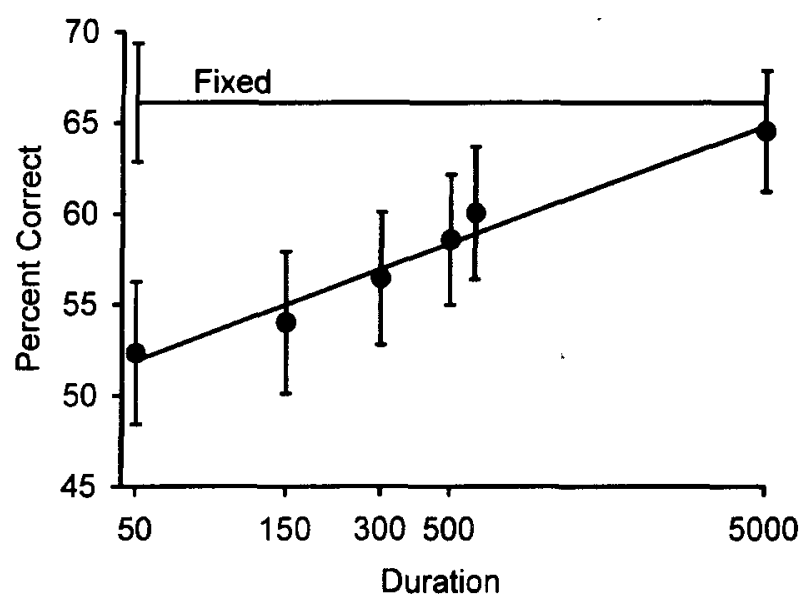

Figure 1. Percent correct identification as a function of the logarithm of the interstimulus interval in the ascending conditions of Experiments 1 and $2(n=40)$. The horizontal line depicts mean percent correct identification in the fixed condition, combining data from both experiments $(n=80)$. iments establish that perceptual interference can be observed with auditory stimuli in much the same way as it is observed with visual stimuli. However, the mechanism underlying perceptual interference may differ across stimulus modalities. This is of particular concern with the temporal manipulations used in the first two experiments because auditory stimuli are inherently temporal, whereas visual stimuli are inherently spatial. Therefore, it is important to determine whether increasing the length of an unfilled interval in the ascending sequence will have a similar effect on picture identification. The third experiment was also a better test of whether a filler task is necessary for the elimination of perceptual interference, because the stimuli for Experiment 3 were drawn from the same source as those from previous work (Snodgrass \& Vanderwort, 1980).

The design of Experiment 3 was very similar to that of the first two, the main differences being the stimulus class and lengths of the ISIs. As in earlier articles on the perceptual interference effect, the stimuli were line drawings taken from Snodgrass and Vanderwort (1980). The line drawings were fragmented using the procedure described by Snodgrass and Hirshman (1991; see Method below). The fixed condition consisted of one exposure to a moderately degraded version of a target object. The ascending condition consisted of three exposures. The first version was very highly degraded, the second one slightly less so. The final version was the same as the single version used in the fixed condition. The interval between exposures in the ascending condition consisted of a blank screen that was maintained for 0,300 , or $1,500 \mathrm{msec}$. The 0 -msec ISI was selected to match the interval typically used in previous experiments, in which one exposure in the ascending sequence was left on screen until the next exposure was presented (i.e., a blank screen did not separate subsequent exposures). The use of a 0 -msec ISI was not possible for the musical stimuli because it would have made distinguishing the boundaries between subsequent exposures very difficult. The remaining two ISIs were selected to represent intervals for which one might $(300 \mathrm{msec})$ and might not $(1,500 \mathrm{msec})$ expect to observe perceptual interference based on the first two experiments. As with the musical stimuli, the competitive activation account predicts that identification performance will vary directly with the length of the ISI and that perceptual interference will be observed at shorter, but not longer, ISIs. In contrast, the blocking hypothesis predicts that identification performance will vary inversely with the length of the ISI and that perceptual interference is more likely to be observed at longer ISIs.

\section{Method}

Subjects. Seventy-two undergraduate volunteers received partial credit toward a course requirement in exchange for their participation and were randomly assigned to one of four counterbalancing orders.

Design. The experiment was a one-way within-subjects design. Presentation style (fixed, ascending - 0 -msec ISI; ascending-300msec ISI; ascending-1,500-msec ISI) was the only independent variable. 
Materials. Sixty line drawings were taken from Snodgrass and Vanderwort (1980); the mean name agreement was $97.7 \%$ ( range = 93-100). The pictures were drawn from a variety of categories, including plants, animals, clothing, furniture, means of transportation, and tools.

The method for fragmenting the images was adapted from that used by Snodgrass and Hirshman (1991). Each image was expanded to occupy a $200 \times 200$ pixel area. A grid consisting of $10020 \times$ 20 pixel squares was placed over each line drawing, and the number of squares containing information (black pixels) was counted. The degraded versions of each line drawing were constructed by randomly deleting a proportion of the squares that contained information. The proportion of information-containing blocks that was retained at each exposure level was calculated using the same algorithm that Snodgrass and Hirshman (1991) used: Prop blocks (level) $=.70^{(8-\text { level })}$. In the present experiment, Levels 1,3 , and 5 were used. Thus, Level 1 retained $.70^{(8-1)}$, or approximately $8 \%$ of the blocks containing information; Level 3 retained approximately $17 \%$ of the information containing blocks, and Level 5 retained approximately $34 \%$ of such blocks. The to-be-deleted blocks were selected randomly and erased by hand. Thus, each subject saw the same degraded images.

The fixed condition consisted of one 2,000-msec exposure to the Level 5 version of a picture. The ascending condition consisted of a 2,000-msec exposure to the Level 1 version, followed by a 2,000msec exposure to the Level 3 version, followed by a 2,000 -msec exposure to the Level 5 stimulus. Successive exposures in the ascending condition were separated by a blank screen that lasted for 0,300 , or $1,500 \mathrm{msec}$. As in Experiments 1 and 2, the last exposure in the ascending condition was identical to the single exposure in the fixed condition.

The line drawings were presented in a different computer-generated random order to each subject. Each line drawing appeared an equal number of times in each condition (fixed and the three ascending conditions) across the experiment.

Procedure. The subjects participated either individually or in groups of 2. Each subject sat at a computer screen. The instructions informed the subjects that they would be asked to identify fragmented versions of common objects. Examples of the fixed and ascending conditions were provided on paper to familiarize the subjects with the types of objects to be named and the approximate amount of fragmentation. None of the examples were included in the set of test stimuli.

The experiment was self-paced. The subjects hit a key on the keyboard to view the next object. After the last exposure in the ascending condition, or the only exposure in the fixed condition, a screen appeared telling the subjects to record their responses on a paper answer sheet. After recording their response, the subjects were instructed to hit a key when they were ready for the next stimulus. A 2,000msec blank screen preceded the presentation of the next stimulus.

\section{Results}

Mean percent correct identification for the fixed condition was $39.5 \%$. Mean percent correct identification for the $0-, 300-$, and $1,500-$ msec ISI conditions was $34.4 \%$, $36.9 \%$, and $36.3 \%$, respectively. As in the first two experiments, identification performance did increase directly with the length of the ISI. However, as in Experiment 2 , the linear trend was not significant $\left[F_{1}(1,71)=\right.$ $\left.0.82, M S_{\mathrm{e}}=.002 ; F_{2}(1,59)=1.27, M S_{\mathrm{e}}=.001\right]$.

Perceptual interference was measured using the same procedures as in Experiments 1 and 2. For both the subject and item analyses, significant perceptual interference was observed at the 0 -msec ISI $\left[t_{1}(71)=2.77, M S_{\mathrm{e}}=\right.$ $\left..018 ; t_{2}(59)=3.21, M S_{\mathrm{e}}=.016\right]$, but not at the $300-\mathrm{msec}$
ISI $\left[t_{1}(71)=1.47, M S_{\mathrm{e}}=.018, p>.40 ; t_{2}(59)=1.47\right.$, $\left.M S_{\mathrm{e}}=.018, p>.40\right]$. The results at the $1,500-\mathrm{msec}$ ISI were inconsistent. Significant perceptual interference was not obtained for the subject analysis $\left[t_{1}(71)=1.96\right.$, $\left.M S_{\mathrm{e}}=.017, .15<p<.20\right]$, but was observed for the item analysis $\left[t_{2}(59)=2.93, M S_{\mathrm{e}}=.013\right]$.

\section{Discussion}

As in the first two experiments, identification performance increased directly with the length of the ISI, at least between 0 and $300 \mathrm{msec}$. In addition, significant perceptual interference was obtained at the shortest ISI, but either did not appear at all, or appeared only inconsistently, at longer ISIs. Once again, this pattern of results is more consistent with the competitive activation framework than it is with the blocking hypothesis.

Although the data were largely consistent with those of the first two experiments, the picture data suggest that perceptual identification dissipates more quickly for visual objects than for musical ones-within about $300 \mathrm{msec}$ as opposed to $500 \mathrm{msec}$. Also, the smooth, linear increase in identification performance over ISIs depicted in Figure 1 was not observed for the line drawings. Identification performance did improve from the 0 -msec condition to the 300-msec condition, but showed no improvement-and in fact showed a very slight, insignificant decrement-from 300 to $1,500 \mathrm{msec}$. These differences will be discussed in more detail in the General Discussion.

\section{GENERAL DISCUSSION}

The experiments reported in this article were designed to answer four related questions. First, will identification performance in an ascending sequence vary directly or inversely with the length of an unfilled interval interpolated between subsequent exposures? Second, will the activation that causes perceptual interference decay on its own, or is some kind of intervening, unrelated activity necessary? Third, will the timing effects observed for auditory stimuli generalize to the visual domain? Fourth, can these data distinguish between accounts for perceptual interference that rely on either consciously generated incorrect hypotheses or transient activation of perceptual structures not associated with the target?

These questions were addressed by manipulating the length of an unfilled ISI between exposures in an ascending presentation sequence. The results were consistent across three experiments using both auditory (familiar melodies) and visual (line drawings) stimuli. As the length of the unfilled interval increased, so did identification performance. For the musical stimuli, a very consistent linear relationship between timing and identification emerged (Figure 1); this same pattern, though somewhat less robust, was also present for the visual stimuli. It is important to mention that the impressive fit depicted in Figure 1 is unlikely to extend far beyond the time frame used in this article. That is, ISIs of less than $50 \mathrm{msec}$ would probably show a much sharper decline in accuracy 
than one would predict on the basis of the regression line shown in the figure, because shorter ISIs would substantially increase the difficulty of determining the boundaries between subsequent exposures in the ascending sequence. Similarly, if the regression line were extended beyond $5,000 \mathrm{msec}$ it would eventually reach a point at which ascending presentation would yield better performance than fixed presentation. This pattern is also unlikely to obtain; $5,000 \mathrm{msec}$ was an extremely long delay relative to the overall pacing of these experiments. Thus, it is unlikely that any significant advantage would be gained by extending the ISI further.

Because there was a direct relationship between identification performance and the length of the unfilled ISI, perceptual interference - poorer identification for an object slowly revealed over multiple trials compared with a single exposure to a moderately degraded version of the object--was observed only at shorter intervals. For visual stimuli, significant perceptual interference was obtained only in the absence of any unfilled interval- that is, when one exposure in the ascending sequence immediately followed another. For musical stimuli, perceptual interference dissipated within approximately $500 \mathrm{msec}$. Thus, the data unambiguously indicate that a filler task, such as the simple addition problem used by Snodgrass and Hirshman (1991), is not required to eliminate perceptual interference. The interfering activation will decay, and quite rapidly, on its own.

Although perceptual interference decayed rapidly for both visual and auditory stimuli, the rate of decay appeared to be steeper for visual stimuli. This difference makes sense given that auditory stimuli in general, and especially the musical stimuli used here, have nonarbitrary temporal structures. The identity of a melody depends on the order and relative timing of successive elements. The same is not true of visual objects like static line drawings, for which identity is determined by the spatial relationships between elements. The different rates of decay for auditory and visual stimuli is also consistent with research on iconic and echoic sensory memory. Typically, visual sensory information (Keele \& Chase, $1967)$ decays more rapidly than auditory sensory information (Darwin, Turvey, \& Crowder, 1972).

As noted, the rapid decay of perceptual interference is much more consistent with Snodgrass and Hirshman's (1991) competitive activation framework than it is with Bruner and Potter's (1964) blocking hypothesis. At the most general level, the two explanations differ with respect to the predicted persistence of the interfering stimulation. The competitive activation framework attributes perceptual interference to the buildup of transient activation, which, presumably, dissipates quickly when initial exposures are removed from view or hearing. In contrast, the blocking hypothesis argues that interference occurs because of the difficulty of deactivating incorrect hypotheses. According to the priming literature, incorrect hypotheses should remain active in memory for much longer than the $300-500 \mathrm{msec}$ interval that eliminated perceptual interference (Reinitz \& Alexander, 1996; Roedi- ger \& McDermott, 1993) even if the distractor object is not consciously activated (Balota, 1983). Thus, competitive activation predicts the rapid dissipation of the effect, whereas blocking does not.

Although the data were consistent with the competitive activation account, the emergence of perceptual interference at only the shortest ISIs in the music experiments leads one to consider whether auditory masking contributed to the observed results. This contention is seriously undermined by the results of Experiment 3, in which an unfilled interval had similar effects on the identification of line drawings (visual masking could not explain these effects, since no interfering visual information ever replaced any portion of the target). Some recently collected data also show that perceptual interference does not obtain when all but the last exposure to a target melody in an ascending sequence is replaced by white noise (Schulkind, 2000). If auditory masking explained the effect obtained here, one would predict that white noise would lead to as much interference as the first few notes of the melody.

There are several other reasons to doubt that auditory masking produced the perceptual interference effects observed in the first two experiments. For example, auditory masking effects generally dissipate completely within $250 \mathrm{msec}$ (Kallman \& Massaro, 1983), whereas the present experiments demonstrated significant perceptual interference, with a 300-msec delay and nonsignificant effects with delays as long as $600 \mathrm{msec}$. Further, because the average note length was approximately $439 \mathrm{msec}$, auditory masking would have interfered with only a relatively small portion of the target: $250 \mathrm{msec}$ (duration of auditory masking) $-50 \mathrm{msec}$ (the shortest ISI in the present article) $=200 \mathrm{msec}$, or slightly less than one half of one note. It is unlikely that the significant effects observed here would result from such a small level of interference. In addition, auditory masking demonstrations usually employ stimuli with minimal context (e.g., isolated tones or words in unrelated lists). The subjects in the present experiments, however, could use the rules of Western music to infer relationships between elements of the melody and/or to predict what might come next. Therefore, one would predict auditory masking to exert a much smaller effect on these stimuli. In fact, everyday speech includes a great deal of auditory masking. However, comprehension of speech streams usually proceeds fluently, in part because of contextual factors (situation, conversation partner, English grammar). Finally, even at the 50-msec ISI, correct identification was quite high (approximately $50 \%$ ), and no subject reported difficulty in parsing the stimulus display.

An interesting direction for future research, and one that should further clarify the mechanism underlying perceptual interference, will be to examine how the cognitive system functions during the ISI. Postexperimental interviews from the present experiments provide some insight. At the end of the experiments, the subjects were asked to describe what they were doing between exposures in the ascending sequence. They reported engaging in a variety 
of behaviors, including (1) rehearsing the most recent exposure of the melody, (2) trying to hum the rest of the melody, and (3) trying to think of the title. The subjects did not report that they found any one of these strategies to be more successful than the others, but an important point can still be drawn from this information. The positive, linear relationship between performance and ISI duration did not result because the subjects made a conscious effort to block out the effects of early exposures. In fact, the subjects were actively attempting to use this information to identify the song. Thus, the release from perceptual interference is probably not mediated by a structure of which the subject is conscious, such as the phonological loop and/or the visuospatial sketchpad of Baddeley's (1990) working memory model.

In sum, the data presented here indicate that the timing of the stimulus display is a critical variable in the emergence of perceptual interference. The effect is observed only when multiple exposures in the ascending sequence follow one another closely in time. Otherwise, perceptual interference decays. The rate at which the effect decays is consistent with the idea that perceptual interference results from transient activation in the perceptual system and that subjects do not have direct control over the part of the system that gives rise to the effect. But, our understanding of the mechanism underlying perceptual interference would be greatly enhanced by future research directed at determining which aspects the stimulus display are responsible for the interference.

\section{REFERENCES}

BADDELEY, A. D. (1990). Human memory: Theory and practice. Boston: Allyn and Bacon.

BALOTA, D. A. (1983). Automatic semantic activation and episodic memory encoding. Journal of Verbal Learning \& Verbal Behavior, 22, 88-104.

BLAKE, R. R., \& VANDERPLAS, J. M. (1950). The effect of prerecognition hypotheses on veridical recognition thresholds in auditory perception. Journal of Personality, 19, 95-115.

Bruner, J. S., \& PotTer, M. C. (1964). Interference in visual recognition. Science, 144, 424-425.

Darwin, C. J., Turvey, M. T., \& Crowder, R. G. (1972). An auditory analogue of the Sperling partial report procedure: Evidence for brief auditory storage. Cognitive Psychology, 3, 255-267.

Frederiksen, J. R. (1967). Cognitive factors in the recognition of ambiguous auditory and visual stimuli. Journal of Personality \& Social Psychology, 7, 1-17.

Frederiksen, J. R. (1969). Response preservation in auditory word recognition. Journal of Experimental Psychology, 79, 48-55.

HANDEL, S. (1993). Listening: An introduction to the perception of auditory events. Cambridge, MA: MIT Press.

HAYS, W. L. (1994). Statistics. New York: Harcourt Brace.

Kallman, H. J., \& Massaro, D. W. (1983). Backward masking, the suffix effect, and preperceptual storage. Journal of Experimental Psychology: Learning, Memory, \& Cognition, 9, 312-327.

Keele, S. W., \& Chase, W. G. (1967). Short-term visual storage. Perception \& Psychophysics, 2, 383-386.

Luo, C. R., \& SNodgrass, J. G. (1994). Competitive activation model of perceptual interference in picture and word identification. Journal of Experimental Psychology: Human Perception \& Performance, 20. 50-60.

PeYNiRCioǦLU, Z. F. (1987). Inhibition through incremental fragment cuing with primed items. Journal of Experimental Psychology: Learning, Memory, \& Cognition, 13, 569-572.

Peynircioǧlu, Z. F., \& Watkins, M. J. (1986). Cue-depreciation: When word fragment completion is undermined by prior exposure to lesser fragments. Journal of Experimental Psychology: Learning. Memory, \& Cognition, 12, 426-431.

Reinitz, M. T., \& AleXANDER, R. (1996). Mechanisms of facilitation in primed perceptual identification. Memory \& Cognition, 24, 129-135.

ROEDIGER, H. L., III, \& MCDERMOTT, K. B. (1993). Implicit memory in normal human subjects. In F. Boller \& J. Grafman (Eds.), Handbook of neuropsychology (Vol. 8., pp. 63-131). New York: Elsevier.

ScHULKIND, M. D. (2000). What causes the interference in perceptual interference? Manuscript in preparation.

SChUlKind, M. D., PosNer, R. I., \& RUBin, D. C. (2000). Musical features that facilitate melody identification: How do you know it's "your" song when they finally play it? Manuscript submitted for publication.

SNODGRASS, J. G., \& HiRSHMAN, E. (1991). Theoretical explorations of the Bruner-Potter (1964) interference effect. Journal of Verbal Learning \& Verbal Behavior, 30, 273-293.

SNODGRASS, J. G., \& VANDERWORT, M. (1980). A standardized set of 260 pictures: Norms for naming agreements, familiarity and visual complexity. Journal of Experimental Psychology: Human Learning \& Memory, 6, 174-215.

\section{APPENDIX}

\begin{tabular}{|c|c|c|}
\hline Song & No. of Notes & $\begin{array}{c}\text { Cumulative } \% \\
\text { Correct }\end{array}$ \\
\hline Amazing Grace & 5 & 60.8 \\
\hline America, the Beautiful & 5 & 57.1 \\
\hline (Oh, My Darling) Clementine & 6 & 53.6 \\
\hline Do-Re-Mi (Do, a Deer) & 5 & 57.1 \\
\hline Frere Jacques & 6 & 53.6 \\
\hline Frosty, the Snowman & 5 & 53.6 \\
\hline Happy Birthday & 4 & 53.6 \\
\hline Home on the Range & 8 & 53.6 \\
\hline I'm Singing in the Rain & 7 & 46.4 \\
\hline I've Been Working on the Railroad & 6 & 32.1 \\
\hline If You're Happy and You Know It & 10 & 53.6 \\
\hline Itsy-Bitsy (Eensy-Weensy) Spider & 9 & 42.9 \\
\hline Joy to the World & 4 & 57.1 \\
\hline Lean on Me & 7 & 42.9 \\
\hline London Bridge Is Falling Down & 5 & 60.7 \\
\hline Mary Had a Little Lamb & 5 & 50.0 \\
\hline National Anthem & 4 & 42.9 \\
\hline On Top of Spaghetti & 6 & 53.6 \\
\hline Pop Goes the Weasel & 6 & 53.6 \\
\hline Puff, the Magic Dragon & 7 & 46.4 \\
\hline Rock-a-Bye, Baby & 4 & 39.3 \\
\hline Row, Row, Row Your Boat & 5 & 53.6 \\
\hline Rudolph, the Red-Nosed Reindeer & 5 & 42.9 \\
\hline Silent Night & 5 & 46.4 \\
\hline Take Me Out to the Ballgame & 6 & 53.6 \\
\hline This Old Man & 5 & 50.0 \\
\hline Three Blind Mice & 6 & 64.3 \\
\hline Tomorrow & 8 & 57.2 \\
\hline Twinkle, Twinkle, Little Star & 4 & 28.6 \\
\hline When You Wish Upon a Star & 6 & 57.1 \\
\hline Yankee Doodle Dandy & 7 & 48.0 \\
\hline Yesterday & 12 & 42.9 \\
\hline
\end{tabular}

Note--The appendix shows the number of notes heard for each song in the article, along with the cumulative probability of identifying the song by that number of notes in previous work (Schulkind et al,, 2000). "I've Been Working on the Railroad" and "Twinkle, Twinkle, Little Star" did not yield any notes within the specified range $(40 \%-65 \%)$, so a value below $40 \%$ was selected.

(Manuscript received June 16, 1998; revision accepted for publication July 16, 1999.) 